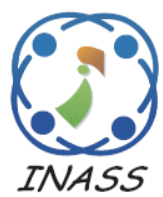

\title{
Achieving Energy Efficiency and Increasing the Network Life Time in MANET through Fault Tolerant Multi-Path Routing
}

\author{
Nanditha Boddu ${ }^{1}$, Ramesh Vatambeti ${ }^{2}$, Veeramallu Bobba ${ }^{3}$ \\ ${ }^{1}$ KL University, Vijayawada, AP, India \\ ${ }^{2}$ IT, Mahatma Gandhi Institute of Technology, Hyderabad, India \\ ${ }^{3}$ Amritasai Institute of Science \& Technology, Vijayawada, India \\ * Corresponding author’s Email: nanditha.boddu@gmail.com
}

\begin{abstract}
A Mobile Ad-hoc network is an autonomous system of mobile hosts connected by wireless links. A MANET has no infrastructure and no centralized administration. The network topology may dynamically change in an unpredictable manner since nodes are free to move. The operation of the nodes in wireless ad-hoc networks depend on the battery power and have limited energy resources. The loss of some intermediate nodes may cause considerable topological changes, weaken the network operation, and have an effect on the lifetime of the network. This makes energy efficiency a key concern in ensuring system resilience. Energy efficient routing problems are important in MANET dynamic environment. Energy should be optimally utilized so that the nodes will perform their action adequately. Fault tolerance is a significant property of ad-hoc network, which assures the reliability of the resources. In this paper, a novel fault tolerant multi path routing protocol is proposed to reduce the packet loss due to route breakage, which uses a new route discovery and maintenance mechanism. It uses alternative route to retransmit the data whenever an intermediate node does not able to forward it, due to link failure or node failure. The proposed protocol is simulated in NS2- and performance is evaluated using packet delivery ratio, end-to-end delay, packet drop, and throughput and energy consumption by varying the pause time, number of flows and traffic rate. Simulation results show that the proposed protocol outperform the existing works in terms of the above metrics.
\end{abstract}

Keywords: Fault tolerance, Residual battery energy, Transmission energy, AOMDV, Node energy level.

\section{Introduction}

In MANET, the operation of nodes depends on battery power and has limited energy resources. This makes energy efficiency a key aspect in ensuring system durability. Further, studies have shown that the communication subsystems consume a large portion of total energy and therefore, solutions for energy conservation are of great significance. Moreover, under some situations, MANET has to be deployed in remote or hostile areas [1].This is impossible to recharge or replace the batteries. Therefore, it is desirable to keep the energydissipation level as low as possible to avoid frequent battery replacement. Energy conservation has posed a big challenge due to MANETs' nature of distributed control, constantly changed network topology and the fact that mobile nodes in MANETs usually are hand-held devices[2][3]. Since a MANET can be quickly and spontaneously arranged, it has intensified attractiveness in scenarios such as disaster rescue operations, battlefields, conferences, etc.[4]

In mobile ad hoc networks, energy efficiency is more important than other wireless networks. As MANET is infrastructure-less network, mobile nodes in ad hoc network must act as a router. Since a MANET is a 'cooperative' network, the nodes join in the process of forwarding packets. Therefore, traffic loads on nodes are heavier than in other wireless networks with fixed access points or base stations [5]. A communication- related energy consumption function is needed to design a system to limit unnecessary power consumption. Energy efficiency design issue must consider the trade- 
offs between different network performance criteria. For example, routing protocols usually try to find a shortest path from a source to a destination. It is likely that some nodes which are on so called 'key positions' will over-serve the network and their energy will be drained quickly, and thus cause the network to 'break'. To avoid this, the energyefficient design should balance traffic load among nodes such that low- power nodes can be idle while traffic is routed through other nodes[6][7]. Since energy is limited in wireless Mobile Ad-hoc Networks, designing energy aware routing protocols has become a main issue. The aim of these protocols is to reduce the energy consumption of the mobile nodes in the network in order to maximize the lifetime of the network [8].

The main purpose of the proposed approach is to maximize the life time of the MANET by conserving the energy of the mobile nodes. This paper is organized in 7 sections. Section 1 and 2 gives the introduction and motivation of the problem. Section 3 gives the related work. Section 4 and 5 describes the proposed algorithm; section 6 illustrates the performance evaluation with other standard protocol. Section 7 presents the Conclusion.

\section{Fault tolerance in MANET}

Most of the existing projects of ad hoc networks depend on the guess associated with a non-opponent environment, that is, each node in the network is cooperative and well-educated. However, in contradictory environment, the nodes of misconduct are always present and the routing performance can worsen considerably.[9] The fault tolerance strategy used in all may prevent the faulty nodes affects all network activity. The fault tolerance will increase the reliability of the system. It may be of different types as follows:

a. Fault tolerance in Node Failures

b. Fault tolerance in Link failure and Network Failure

c. Fault tolerance in Transmission Power and Energy

d. Fault tolerance using check-pointing, message logging, reducing overload etc.

The fault nodes in a mobile ad hoc network (MANET) reduces the functionality of routing protocol. The use of greedy routing mechanisms in which every time go to one path, can lead to considerable data loss, if there is a failure of this route in a fault-prone environment. However, the use of all available routes leads to an undesirable amount of overhead in the network. The development of an efficient routing protocol and fault tolerant inherently complicated.

\section{Related work}

Saravanan N et al (2016) [8] proposed Mobile Agent based Energy Efficient Reliable routing protocol for MANET for reliability and energy efficiency. The link cost metric is evaluated by means of Network Load in terms of node burthen degree, bandwidth usable degree, Minimum Drain Rate (MDR) for energy consumption and Link availability. Then based on the collected information's a combined list cost metric is estimated. As a final point, after collecting information's from agents multiple paths is established and then the source selected the optimal path using the path cost metric.

V. Jayalakshmi et al (2012) [10] proposed an energy efficient multipath fault tolerant routing protocol to improve reliability and improve the routing stability in mobile ad hoc networks. The proposed approach is a multi-target routing protocol that satisfies various application requirements, taking into account the network conditions, compared with some stable and routing protocols based on representative sources such as SMS SMR. There has been a noticeable improvement in the package delivery ratio and also the extreme delay of the decline of extreme contrast to the different protocols.

RoieMelamed et al (2008) [11] present a faulttolerant and efficient position-based routing protocol for large MANETs called Octopus. Fault-tolerance is accomplished by using redundancy, i.e., keeping the position of each node at various nodes, and also by maintaining often restored soft state.

Karim Khazaei et al (2009) [12] present a new scheme tolerant site management error called FTLM, which is based on us. With the site management scheme, each source node within the network is based on a smaller subset associated with us, the location server and to change the with the new geographic location. The fault tolerance in this strategy is achieved by redundancy in site servers that store the node locations. FTLM is better scalable than to compare Octopus and some other site management methods.

Olufemi Adeluyi et al (2012) [13] proposed a new routing algorithm for MANETs inspired by nature- Spiral Millipede-inspired Routing Algorithm (SMiRA). It is designed as resource light techniques that minimize routing overhead as well as enhances the degree of tolerance to routing faults, among 
other things. It was compared to a standard MANET routing protocol called AODV and the result shows significant improvement in the following areas: Routing overhead, Fault Recovery Latency .

John Oommen B et al (2010) [14] The faultprone nodes, employing all the accessible paths which produce an unwanted amount of overhead on the system. .To handle the issue of effective faulttolerant routing, present a fault tolerant routing algorithm (FTAR), using concepts of how swarms of natural ants work. The algorithm is break into different phases specifically initialization, path selection, pheromone deposition, confidence calculation, evaporation and negative reinforcement. FTAR better than leading fault-tolerant MANET routing protocol at present, based on the amount of routing overhead.

Kiran $\mathrm{K}$ et al (2014) [15] discusses fault tolerance in a multi-radio network. The fault tolerance can be executed when the routing algorithm uses Beehive. The multi-radio nodes are equipped with WiMAX and Wi-Fi. The article talks about fault tolerance that is used in multi-radio, us, which are used for the traffic transfer in the data transmission. One method is not to handle systematic errors in the hardware radios through the traffic sharing use and to unite with the BeeHive routing algorithm. Tolerance band allows the transmission of continuous data and disaster recovery mechanisms.

\section{Energy efficient algorithm for increasing network life time}

The objective of the proposed algorithm is to increase the network life time by minimizing the total transmission energy using energy efficient routes to transmit the packet. The proposed algorithm has three main steps.

Step 1: Calculating Transmission Energy

The transmission energy (TEnode) of each node relative to its distance with another node is calculated by using Eq.(1)

$$
\begin{aligned}
& T E_{\text {node }} \alpha d^{n} \\
& T E_{\text {Node }}=k d^{n}
\end{aligned}
$$

where $\mathrm{k}$ is constant and $\mathrm{n}$ is path loss factor which is generally between (2-4).

Step 2: Selection Criteria:

The node must have more residual battery power (RBE) than the required transmit power (TEnode) to transmit the packet to the nearest node in the route. All nodes on the route will be checked with this condition, even if a node of a route does not meet this condition, this route is not considered a viable solution. All other routes to all nodes with a sufficient amount of energy is considered a viable solution. And those nodes having equal RBE than (TEnode ) are made to go into sleep mode. This selection criterion helps to extend the life of the network while avoiding breaking the connection. We try to avoid the repeated use of the paths. But at a stage, we need to commit energy efficiency when we make a route with less power consumption but is already used and a defeat with maximum power consumption that is not used. Therefore, at this point, we avoid the repeated use of the paths and try to extend the life of the network. Transmission energy of a node to node in a rout is calculated according to the distance and the total transmission energy (TTER) for that rout is calculated using Eq. (2).

$$
T T E R=\sum_{i=1}^{M} T E
$$

where $\mathrm{m}$ is the number of hops in the route, TE $=$ TEnode is the transmission energy between the nodes. The route with minimum total transmission energy i.e. min (TTER) will be selected as energy efficient route.

Step 3: Calculating Residual Battery Energy (RBE):

After transmitting the packet, residual battery energy for each node of the route is calculated using Eq. (3) with parameters initial battery energy (IBE) and TEnode.

$$
R B E=I B E-T E_{\text {node }}
$$

\subsection{Pseudo code}

Step 1: Generate all the possible routes.

Step 2: Calculate the TEnode for each node of each route using eq. (1).

Step 3: Check the below condition for each route till no route is available to transmit the packet.

$$
\text { if }\left(R B E \leq T E_{\text {node }}\right)
$$

Make the node into sleep mode. Else

Select all the routes which have active nodes

End

Step 4: Calculate the total transmission energy for all the selected routes using eq. (2).

Step 5: Select the energy efficient route on the basis of minimum total transmission energy of the route. 
Step 6: Calculate the RBE for each node of the selected route using eq. (3).

Step 7: go to step 3.

Step 8: End.

\section{Fault tolerant multi-path routing protocol}

In Ad-hoc On-demand Multi-path Distance Vector routing (AOMDV), it determines multiple paths by broadcasting RREQ packets. However, the multiple routes have been determined according to the time when the RREQ packet arrived. It does not consider the energy in the paths. The proposed approach not only considers residual energy in paths selection but also the energy balance in data transmission to increase the lifetime of the network. The battery power $E_{b}$ is given by

$$
E_{b}=\sum_{i=1}^{n} B_{i}
$$

Where $B_{i}$ is the individual battery of the node. $E_{a v}$, is the average energy of the nodes. It is given by

$$
E_{a v}=\frac{\sum_{i=1}^{n} E_{r}}{n}
$$

Where $E_{r}$ is the residual energy of node $i$ and $n$ is the number of nodes along the path. Now the energy level of node $E_{e l}$ is given by

$$
E_{e l}=\frac{E_{r}}{E_{a v}}
$$

The smaller the value of the battery power, better the path runs.

\subsection{Algorithm}

Consider residual energy and battery power in paths selection and the energy balance in data transmission to increase the lifetime of networks. Let $\mathrm{A}$ and $\mathrm{C}$ be the source and destination nodes.

1. Calculate battery power $\mathrm{E} b$

2. Compute the average energy of the nodes E $a v$

3. Calculate the Energy level of node Eel

4. RREQ mod ified $=(\mathrm{RREQ}+(\mathrm{E} b+\mathrm{E} a v))$

5. If $\mathrm{S}$ requires a route to destination, then

5.1 Check routing table

5.2 If Path is invalid, then

5.2.1 S performs route discovery (with network-wide flood of RREQ)

End if

End if

6. If node receives RREQ, then
6.1 If RREQ is from D and RREQ has route to the $\mathrm{D}$, then

6.1.1. Stores the first received RREQ in buffer

6.1.2. Starts the timer

Else

6.2 Process proceeds as conventional AOMDV

End if

7. If Node receives other copies of RREQ, then

7.1 If RREQ provides new disjoint path, then

7.1.1RREQ is stored in the buffer of the node.

Else

7.1.2 Dropped.

End if

7.2 If timer expires, then

7.2.1 Node drops all copies of RREQ

End if

7.3 If Battery power is minimum

7.3.1 The destination node replies with $k$ copies of RREQ in buffer

E nd if

End if

8. If intermediate node does not have valid route to destination, then

8.1 forward fist received RREQ.

End if

9. If Eel> Eth

9.1 intermediate nodes forward the RREQ. Else

9.2 drop RREQ

10. Calculate received signal strength $\mathrm{P} R$ using (3.4)

11. If $P R T \mathrm{~min}$

11.1 Node $C$ is about to fail shortly

11.2 Node D informs node B about status of node $\mathrm{C}$

11.3 B starts caching the data packets in its data buffer

End if

12. If $P R T \mathrm{~min}$,

12.1 Node $\mathrm{C}$ is completely failed

12.2 Node D informs node B about the status of node $\mathrm{C}$

12.3 Node B salvages all packets that are still in its data cache through the established alternate path.

End if

\section{Simulation results}

NS2 is used to simulate the proposed protocol. In this experiment, 50 mobile nodes move in a 1500 meter $x 300$ meter region for the simulation time of 100 seconds. Each node moves independently with the same average speed. All nodes have the same transmission range of 250 meters. In the simulation, 
the speed is set as $5 \mathrm{~m} / \mathrm{s}$. The simulated traffic is Constant Bit Rate (CBR). The pause time is varied as $0,10,20,30,40$ and 50 seconds for the mobile nodes. The proposed approach is compared with the AOMDV. In this simulation, the Pause time is varied as $0,10,20,30,40$ and 50 secs.

Figure 1 presents the packet delivery ratio of both the protocols. When the pause time is increased, the node mobility will be decreased and hence link breakages and network disconnections will be reduced. So the packet delivery ratio increases, as the pause time is increased from 0 to 50 seconds. From the figure, it is seen that FTMPR achieves 5\% higher delivery ratio, compared to normal AOMDV, since FTMPR choose fault tolerant multiple paths for data transmission.

Figure 2 presents the end-to-end delay of both the protocols. When the pause time is increased, the node mobility will be decreased and hence link breakages and network disconnections will be reduced. So the delay involved in rerouting and route maintenance will be minimized. So the delay slightly decreases, as the pause time is increased from 0 to 50 seconds. From the figure, it is seen that the average end-to-end delay of the proposed FTMPR protocol is $45.5 \%$ less when compared to the AOMDV protocol, since it proactively reduces the path failures.

Figure 3 presents the received throughput of both the protocols. When the pause time is increased, the node mobility will be decreased and hence link breakages and network disconnections will be reduced. So the throughput increases, as the pause time is increased from 0 to 50 seconds. As it is seen from the figure, the throughput is $5.8 \%$ more in the case of FTMR, than AOMDV, since FTMPR choose fault tolerant multiple paths for data transmission.

Table 1. Simulation Parameters

\begin{tabular}{|l|l|}
\hline No. of Nodes & 50 \\
\hline Area Size & 1500 X $300 \mathrm{~m}$ \\
\hline Mac & 802.11 \\
\hline Radio Range & $250 \mathrm{~m}$ \\
\hline Simulation Time & 100 seconds \\
\hline Traffic Source & CBR \\
\hline Packet Size & 512 bytes \\
\hline Mobility Model & Random Way Point \\
\hline Pause time & $0,10,20,30,40$ and 50 seconds \\
\hline
\end{tabular}

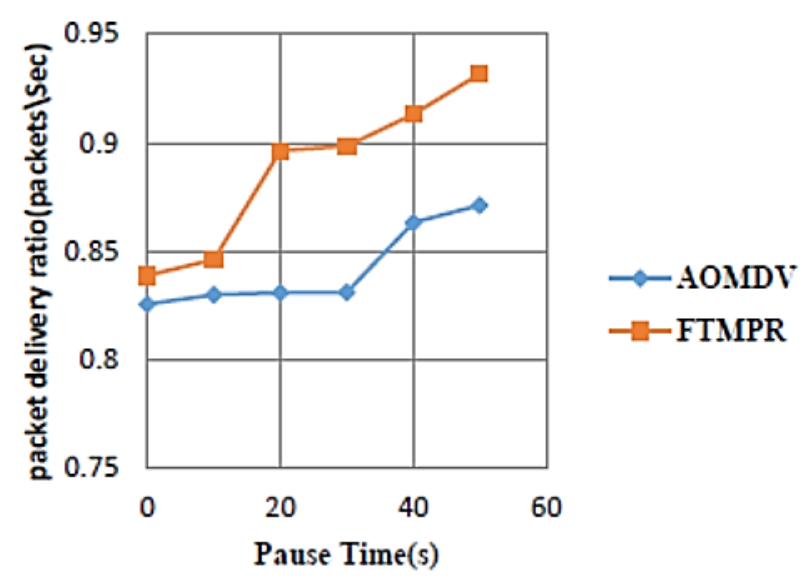

Figure.1 Packet Delivery Ratio Vs Pause Time

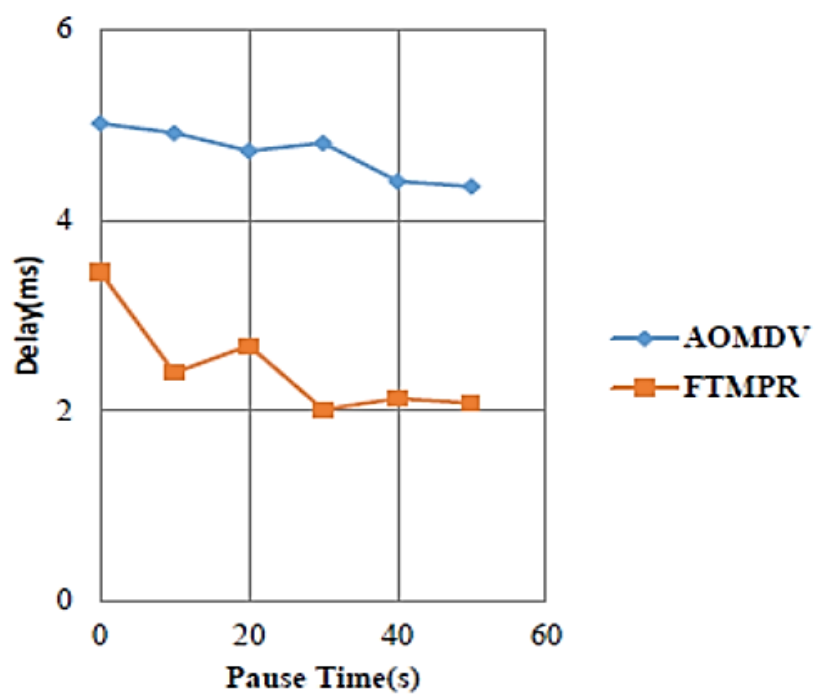

Figure.2 Delay Vs Pause Time



Figure.3 Throughput Vs Pause Time

Figure 4 presents the average packet drop occurred for both the protocols. When the pause time is increased, the node mobility will be decreased and hence link breakages and network disconnections will be reduced. So the packet drop decreases, as the pause time is increased from 0 to 


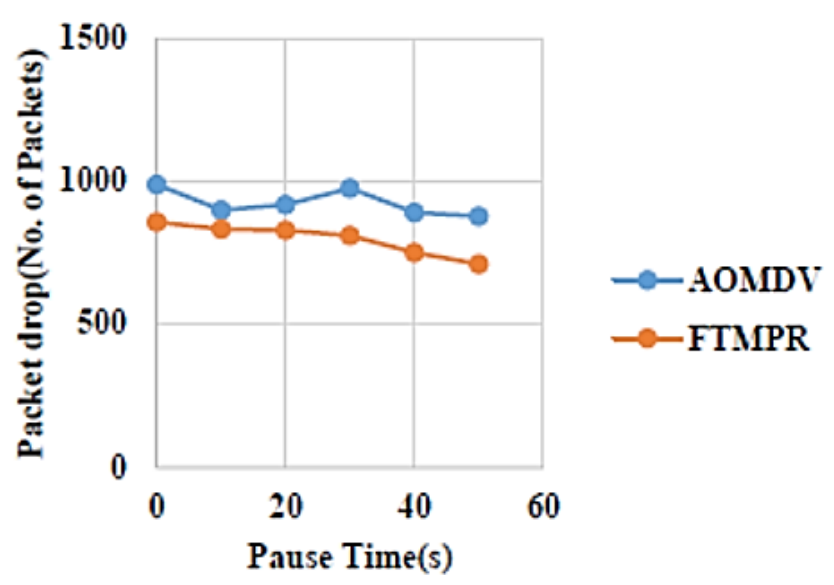

Figure.4 Packet Drop Vs Pause Time

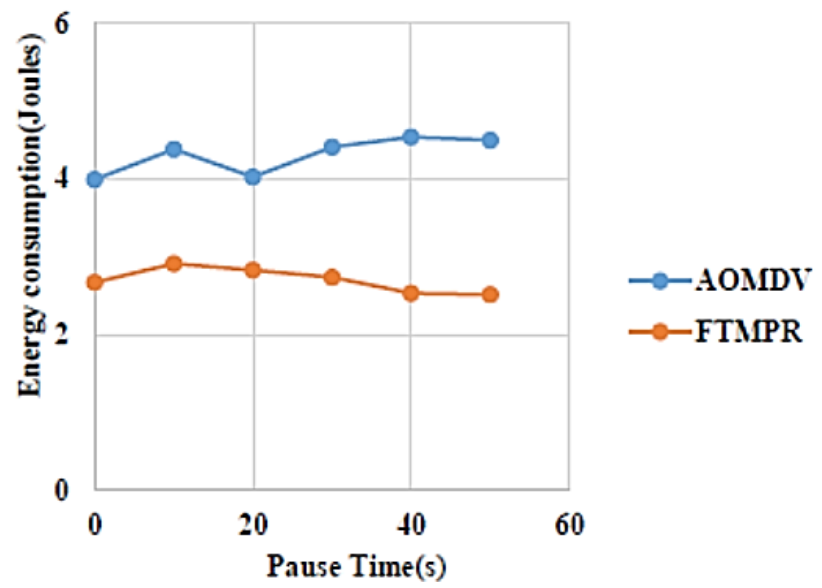

Figure.5 Throughput Vs Pause Time

50 seconds. From the figure, it is ensured that the packet drop is $13.6 \%$ less for FTMPR when compared to AOMDV, since it proactively reduces the path failures.

Figure 5 shows the average energy consumption of both the protocols. When the pause time is increased, the node mobility will be decreased and hence link breakages and network disconnections will be reduced. So the energy consumption is decreased when the pause time is

increased. Since FTMPR selects energy efficient routes, it consumes average of $37.13 \%$, less energy than AOMDV.

\section{Conclusion and Future work}

In this paper, the proposed fault tolerant multi path routing protocol is simulated to study its performance. To reduce the packet loss due to route breakage, a new route discovery mechanism has been used. In this protocol, nodes determine multiple disjoint routes with more battery power and residual energy, to every active destination. When a downstream node encounters a forwarding error, an upstream node with the same data in its buffer and alternative route can retransmit the data. In the faulttolerant mechanism, the received signal strength is measured and based on its value it can send warning packets to the previous node. The AOMDV protocol is used as a base for the multipath routing. The proposed scheme enables more nodes to salvage a dropped packet, (i.e.) packet salvaging is distributed. From simulation results, it is evident that the proposed protocol achieves better throughput and packet delivery ratio with reduced delay, packet drop and energy. The proposed scheme FTMPR achieves 5\% higher packet delivery ratio, $45.5 \%$ lesser average end-to-end delay, 5.8\% higher throughput, $13.6 \%$ lesser packet drop and $37.13 \%$ lesser energy consumption when compared with AOMDV.

As a future work we shall consider reducing overhearing during route discovery for optimized energy management.

\section{References}

[1] E. R. Ahmed, "A fault-tolerant routing protocol for mobile ad hoc networks", Journal of Advances in Information Technology, Vol. 2, No.2, pp.128-132, 2011.

[2] A. Malek, Li. Chunlin, Z. Yang, A.H. Naji Hasan, and X. Zhang, "Improved the Energy of Ad hoc On- Demand Distance Vector Routing Protocol", In: Proc. of the International Conference on Future Computer Supported Education, pp. 355-361, 2012.

[3] S. Jain and S. Jain, "Energy Efficient Maximum Lifetime Ad-Hoc Routing (EEMLAR)", International Journal of Computer Networks and Wireless Communications, Vol.2, No.4, pp. 450-455, 2012.

[4] G. Nagaraja and P. R. Ch, "A Reliable Group Key Management Framework Using Fuzzy Logic for MANETs", International Journal of Intelligent Engineering and Systems, Vol.9, No.4, pp. 107-115, 2016.

[5] P. Manickam and D. Manimegalai, "A highly adaptive fault tolerant routing protocol for energy constrained mobile adhoc networks", Journal of Applied and Theoretical Information Technology, Vol.57, No.3, pp.388-397, 2013.

[6] P.H. Le, "A Performance Evaluation of Multipath Routing Protocols in Mobile Ad hoc Network", In: Proc. of IEEE 15th International Conference on Computational Science and Engineering, pp. 67-75, 2012. 
[7] S. Mishra and S.M. Satpathy, "Energy efficiency in ad hoc networks", International Journal of Ad hoc, Sensor \& Ubiquitous Computing, Vol.2, No.1, pp-139-145, 2011.

[8] S. Nallusamy, S. Appavupillai, and S. Ponnusamy, "Mobile Agents based Reliable and Energy Efficient Routing Protocol for MANET", International Journal of Intelligent Engineering and Systems, Vol.9, No.3, pp. 110116, 2016.

[9] W. Li, D. Liu, and H. Zhao, "Fault-Tolerance Mechanism of Mobile Agent In Mobile Ad Hoc Networks", In: Proc. of the Wireless Communications, Networking and Mobile Computing, 2008. WiCOM'08. 4th International Conference on, pp. 1-4, 2008.

[10] V. Jayalakshmi and R.R. Kumar, "Multipath fault tolerant routing protocol in MANET", International Journal on AdHoc Networking Systems, Vol.2, No.1, pp.23-35, 2012.

[11] M. Roie, I. Keidar, and Y. Barel, "Octopus: A fault-tolerant and efficient ad-hoc routing protocol", Wireless Networks, Vol. 14, No. 6, pp. 777-793, 2008.

[12] K. Khazaei, S. Mohammadi, and A. Momeni. "A fault tolerant location management for MNANET", In: Proc. of the Wireless and Microwave Technology Conference, 2009. WAMICON'09. IEEE 10th Annual, pp. 1-6, 2009.

[13] A. Olufemi, and J.A. Lee, "SMiRA: A bioinspired fault tolerant routing algorithm for MANETs", In: Proc. of the ICT Convergence (ICTC), 2012 International Conference on, pp. 78-84, 2012.

[14] B.J. Oommen and S. Misra, "Fault-tolerant routing in adversarial mobile ad hoc networks: an efficient route estimation scheme for nonstationary environments", Telecommunication Systems, Vol.44, No. 2, pp.159-169, 2010.

[15] K. Kiran, P.D. Shenoy, K.R. Venugopal, and L.M. Patnaik, "Fault tolerant BeeHive routing in mobile ad-hoc multi-radio network", In: Proc. of the Region 10 Symposium, 2014 IEEE, pp. 116-120, 2014.

[16] J. SA, A. Bande, G. Deshmukh, Y. Rade, and M. Sandhanshiv, "An improvement in congestion control using multipath routing in MANET", International Journal of Engineering Research and Applications, Vol.2, No.3, pp.509-514, 2012.

[17] C. Priyadharshini, and K. ThamaraiRubini, "Predicting route lifetime for maximizing network lifetime in manet", In: Proc. of the International Conference on Computing, Electronics and Electrical Technologies, pp.792-797, 2012.

[18] S.M. DilipKumar and B.P.V. Kumar, "EnergyAware Multicast Routing in MANETs: A Genetic Algorithm Approach', International Journal of Computer Science and Information Security, Vol. 2, No.2, pp. 81-90, 2009.

[19] K. Arulanandam and B. Parthasarathy, "A New Energy Level Efficiency Issues in MANET", International Journal of Reviews in Computing, Vol. 2, No.4, pp. 43-52, 2009. 